\title{
Mechanical behaviour at large strain of polycarbonate nanocomposites during uniaxial tensile test
}

\author{
A. Christmann, P. Ienny, J.C. Quantin, A.S. Caro-Bretelle*, J.M. Lopez-Cuesta \\ Ecole des Mines d'Alès, 6 Avenue de Clavières, 30319 Alès, France
}

\section{A B S T R A C T}

\begin{abstract}
The present study focuses on the mechanical behaviour of polycarbonate nanocomposites reinforced by alumina or silica nanoparticles at low levels of incorporation. More particularly, we present an experimental approach, specific to large strain measurements by using a Digital Image Correlation (DIC) technique. The mechanical mechanisms involved during uniaxial tensile test of polycarbonate nanocomposites were studied at two-dimensional scales. First, elastic properties of the nanocomposites were determined at macro-homogeneous scale and compared to values obtained by continuum-based elastic micromechanical models. Then the in-plane kinematics measurements at the central part of a doubleedge notched sample is analysed locally. The evolution during the test of the volumetric strain and axial strain profiles were studied before and after the yield stress: this analysis revealed the existence of several damage processes during the test up to rupture and put in relief the influence of fillers. Finally, the necking phenomenon was statistically studied and the shape of the neck in terms of strain intensity and localisation was analysed.
\end{abstract}

Keywords:

Nanocomposites

Polycarbonate

Digital image correlation

\section{Introduction}

The use of nanoparticles as reinforcements for polymer matrices is relatively recent and is due to their ability to impart strong improvements of performances at very low volume fractions in comparison to micrometric particles. Nanocomposites find applications in various fields as their enhanced properties can be mechanical, electrical, optical, ... [1-9].

Particularly, nano-fillers are widely used to provide mechanical reinforcement to the polymer matrix $[10,11]$. The experimental investigation of such materials consists in several approaches according firstly to the scale of observation and secondly to the confrontation between different phenomenological mechanisms during the mechanical solicitation. Numerous studies deal with elaboration and evaluation of mechanical properties of nanocomposites but, most of the time, only macroscopic properties are evaluated. At a macroscopic scale, it appears that incorporation of nanoparticles into a polymeric matrix influences elastic modulus but the influence of the nanoparticles on local reinforcement mechanisms has hardly been studied [12-15].

\footnotetext{
* Corresponding author. Tel.: +33 466785631; fax: +33 466785680. E-mail address: anne-sophie.caro@mines-ales.fr (A.S. Caro-Bretelle) URL: http://www.ema.fr
}

Nevertheless, micromechanisms of deformation of each phase, decohesion between matrix and fillers or cavitation phenomena inside fillers produce inhomogeneity of deformation and thus are responsible of microvoids creation and crazing [16]. Like several other engineering polymers, polycarbonate undergoes, during uniaxial tensile tests, an inhomogeneous localized deformation at rather low strains; the phenomenon is called necking and is characterized by a section reduction of the tensile bar. Standardized mechanical tensile tests are unsuitable to describe these micromechanisms as they only provide the average strain over the utile length. In this work we propose to use a non-contact measurement technique which is more and more appealing in experimental mechanics. Digital Image Correlation (DIC) offers a good compromise to describe both the macro-homogeneous material response and the non-homogeneous strain induced by a higher strain level from the same mechanical test $[13,17,18]$. This technique allows measuring the displacement fields at the sample's surface. Under the assumption of transversal isotropy, it is then possible to calculate local volumetric strain all over the investigated zone of the sample during the tensile test. If this technique, used for about ten years, has already provided interesting data on damage mechanisms of unfilled polymers, such as PC, PMMA [19-21], the present work investigates the effects of nanoparticles on the neck initiation and propagation.

The present study focuses on the mechanical behaviour at macroscopic and mesoscopic scales of polycarbonate 
Table 1

Materials.

\begin{tabular}{llllll}
\hline Sample & PC & Alu C 1\% & Alu C 3\% & A300 1\% & A300 3\% \\
\hline Filler & - & Alumina & Alumina & Silica & Silica \\
$\begin{array}{c}\text { Incorporation } \\
\text { rate (wt\%) }\end{array}$ & - & 1 & 3 & 1 & 3 \\
\hline
\end{tabular}

nanocomposites reinforced by alumina or silica nanoparticles at low volume fractions. More particularly, by using DIC, the mechanical mechanisms involved during uniaxial tensile tests of polycarbonate nanocomposites were studied. The first section is devoted to the description of the displacement field measurement method, at macro and meso scales. Setting-up of a methodology to evaluate the two-dimensional fields of in-plane displacement is described and issued from a numerical calibration. In practice, outof-plane motions are unavoidable, like Poisson's effect, deviation of the grips and more especially when necking occurs during the loading process. In this study a great attention was turned on the choice of correlation parameters to minimize error in measurements and estimate their effect on the strain tensor calculated locally. The second part of this paper deals with the characterization of macro-homogeneous properties: results are then compared to values obtained by continuum-based elastic micromechanical models. The evolution of local strains is investigated in the third part and axial strain profiles are analysed before and after the neck initiation. The volumetric strain is studied at the cross-section where the necking dominates. The damaging processes were studied as a function of formulation. The results obtained in this study show how fillers bring significant differences in the onset and propagation of necking. Finally, a statistical study of the necking phenomenon in terms of strain intensity and localization is presented. A short discussion about the event-character of both the onset but also the propagation of necking concludes the paper in the last part.

\section{Experimental}

\subsection{Materials under study}

The polycarbonate used in this study is a poly(bisphenol Acarbonate) supplied by Bayer under the trade mark Makrolon ${ }^{\circledR}$ AL2647. Polycarbonate pellets were dried in a vacuum oven at $120{ }^{\circ} \mathrm{C}$ for a minimum of $12 \mathrm{~h}$ prior to the compounding.
Two kinds of spherical nanoparticles were selected for this study:

-AEROXIDE ${ }^{\circledR}$ Alu C (Degussa). It is a highly dispersed fumed metal oxide mainly composed of alumina $\left(\mathrm{Al}_{2} \mathrm{O}_{3} \mathrm{wt} \%>99.8\right)$. The specific surface area (BET) is $100 \pm 15 \mathrm{~m}^{2} / \mathrm{g}$. The average primary particle size is $13 \mathrm{~nm}$;

-AEROSIL ${ }^{\circledR}$ A300 (Degussa). It is a hydrophilic fumed silica mainly composed of silicaa $\left(\mathrm{SiO}_{2} \mathrm{wt} \%>99.8\right)$. The specific surface area (BET) is $300 \pm 30 \mathrm{~m}^{2} / \mathrm{g}$. The average primary particle size is $7 \mathrm{~nm}$.

The fillers are used without any coating or coupling agent and were dried for $6 \mathrm{~h}$ before mixing with the polycarbonate.

The nanocomposites were compounded in an intermeshing corotating twin-screw extruder Clextral BC 21 (screw diameter $25 \mathrm{~mm} ; L / D=36$ ). The temperature along the screw was controlled at $280{ }^{\circ} \mathrm{C}$ and the extrudate was pelletized. The pellets were dried under vacuum at $120^{\circ} \mathrm{C}$ for a minimum of $12 \mathrm{~h}$ and then injection moulded at $280^{\circ} \mathrm{C}$. The mould temperature was kept at $100{ }^{\circ} \mathrm{C}$.

The moulded samples are tensile bars corresponding to the ISO 527-2 1-A type standard.

Five different formulations were thus realized and are presented in the Table 1.

\subsection{Testing apparatus}

\subsubsection{Mechanical testing}

Uniaxial tensile tests were conducted on a Zwick TH010 universal testing machine according to the ISO 527 standard. The crosshead speed is equal to $1 \mathrm{~mm} / \mathrm{min}$. The tensile test is conducted up to the rupture of the specimen. The software used is TestXpert ${ }^{\circledR}$ and allows the recording of time, load and elongation. Let's define by $y$ the tensile direction, $x$ perpendicular to $y$ and in the planar surface and $z$ the out-of-plane direction.

The nominal stress is defined by the following expression: $\sigma_{N}=F / S_{0}$ where $F$ is the recorded load and $S_{0}$ the initial sample section surface.

\subsubsection{Optical testing}

2.2.2.1. Apparatus. The optical extensometer involves a high resolution Charge-Coupled Device (CCD) camera (Redlake Megaplus II, $1920 \times 1080$ contiguous and square pixels, coded in 256 grey a

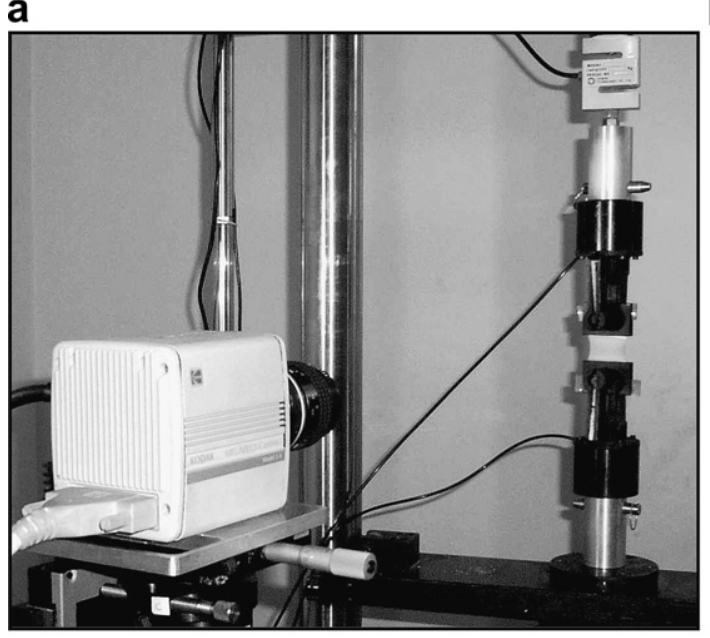

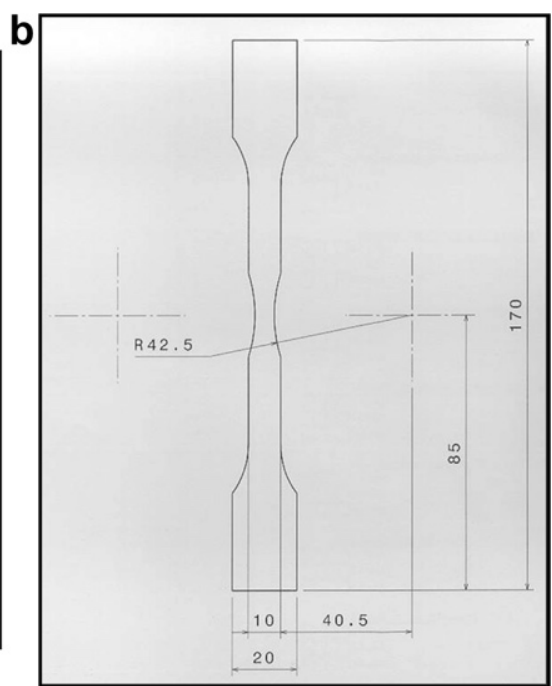

Fig. 1. Testing apparatus (a) Geometry of the specimen (b). 
a

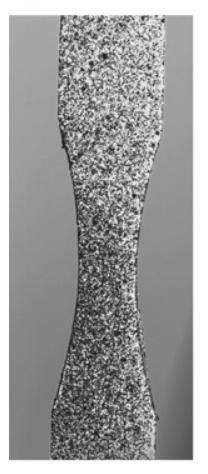

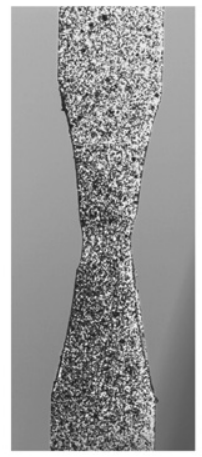
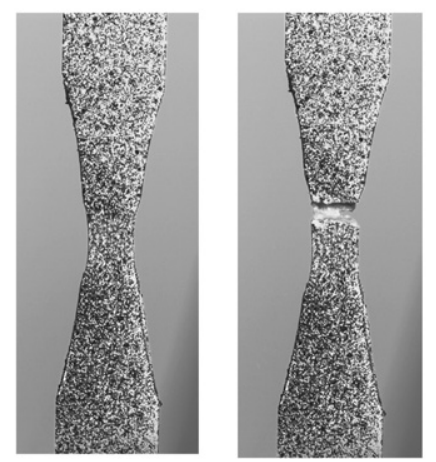

b

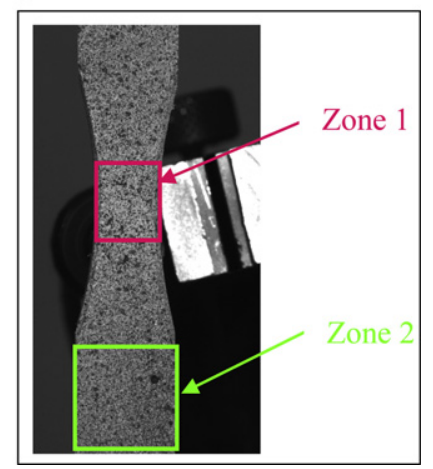

Fig. 2. Sequence of images (a) Zones of Interest (ZOI) (b).

levels), set in front of the specimen, which records images during the test Fig. 1(a). The optical axis of the camera remains perpendicular to the in-plane surface of the specimen during the test. The images acquisition is commanded by a LabVIEW ${ }^{\circledR}$ software which allows the simultaneous acquisition of the images and the data from the testing machine (such as load and crosshead displacement). According to the test speed used, images are recorded every $2 \mathrm{~s}$. The scale factor is fixed to $42 \mu \mathrm{m}$ per pixel.

2.2.2.2. Sample preparation. In order to study the necking phenomenon during the tensile test, tensile bars were machined so that the neck initiation takes place within the observation field of the camera. A curved profile was created in the central area as illustrated in Fig. 1(b) where the data are in millimetres and $\mathrm{R}$ is the radius of curvature. The same type of specimen was used for all tests. Given that the creation of this curved profile induces stress heterogeneities over the section and in order to keep the tensile load quite uniaxial, the radius of curvature was fixed so that this stress heterogeneity remains inferior to $5 \%$.

A random speckle pattern was applied to each specimen with firstly a uniform primary white paint layer and secondly a speckle of blue paint blobs. Both paints are sprayed on the sample surface, so the solvents (isopropyl alcohol) are very quickly eliminated and are supposed to induce no swelling of the polymer. This creates the image signature. Fig. 2(a) presents a selected sequence of images from unloaded specimen to failure.

Displacement fields were evaluated in two different areas of the tensile bar: in the central zone (Fig. 2(b) Zone 1), where strain localization phenomena takes place, and in the non-machined zone (Fig. 2(b) Zone 2). The elastic properties were particularly calculated in the non-machined zone far away from local instabilities.
2.2.2.3. Digital Image Correlation (DIC) processing. The image processing, carried out after the test, is based on a direct image correlation computation, leading to subpixel accuracy on the twodimensional displacement components. Among commonly used correlations functions, the normalized discrete inter-correlation function was used in the present work. A subpixel resolution is obtained by a local interpolation performed in the vicinity of the discrete maximum of the correlation map. From two treated images, a correlation calculation is made at every meshing point (Fig. 3). Each point of the virtual mesh corresponds to the centre of a pattern. This pattern, defined by its size denoted CS (for correlation size), is the representative area of the material point which is tracked. The mesh corresponds to the Gage Lengths (GL) where average values of the strain tensor components are investigated. The mesh is defined by the number MS of points (MS for Mesh Size) which can be taken identically or not according to both directions, and by the distance Gs between two points (Gs for Grid Step).

The digital image processing leads to an incremental displacement field. One has then to sum the displacement components to achieve the total displacement.

The distance between the processed image and reference image does not exceed 20 increments for a sequence of 100 acquired images because of a correlation calculation called "mixed processing": acquired images are arranged in successive groups of 12 images, each image being correlated with the first image of the group it belongs (Fig. 3(a)). For a given image, the displacement field is obtained by summing the cumulative displacements evaluated successively for each group and the displacement calculated for the group to which the image belongs. The choice of the number of images per group is limited by the maximum strain increment calculated by correlation in the necking zone [13].

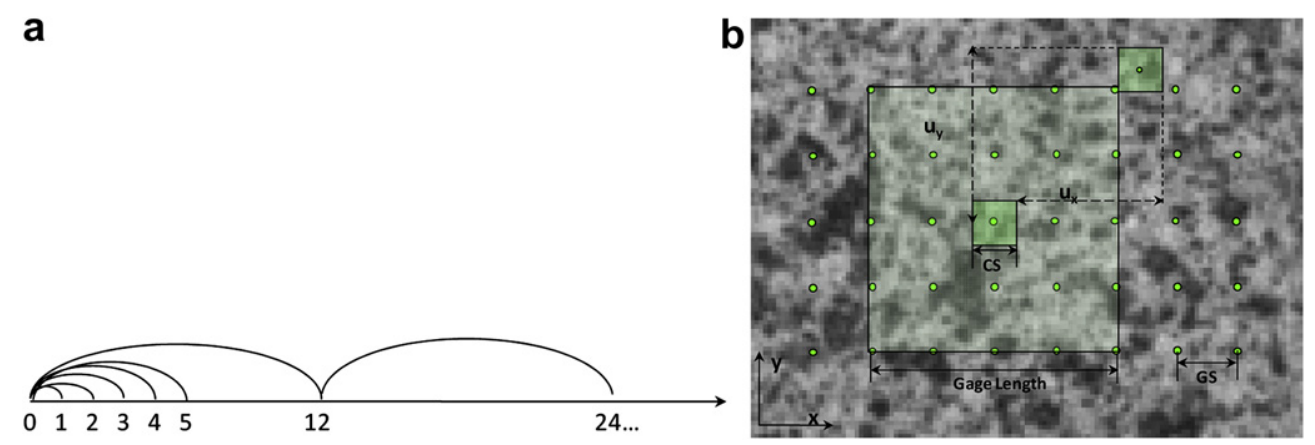

Fig. 3. Definition of the two-dimensional digital extensometer (CS: correlation size; GS: grid step); “mixed processing” (a) Gage Length (b). 
Table 2

Data parameters used by the DIC Process.

\begin{tabular}{|c|c|c|c|}
\hline \multirow[b]{2}{*}{ Mesh Size (line by colon) } & \multirow{4}{*}{$\begin{array}{l}\text { (pixels) } \\
(\mathrm{mm})\end{array}$} & \multirow{2}{*}{$\frac{\text { Zone } 1}{50 \times 39}$} & \multirow{2}{*}{$\frac{\text { Zone2 }}{5 \times 5}$} \\
\hline & & & \\
\hline \multirow[t]{2}{*}{ Gs } & & 5 & 20 \\
\hline & & 0.21 & 0.84 \\
\hline \multirow[t]{2}{*}{ Cs } & (pixels) & 30 & 30 \\
\hline & $(\mathrm{mm})$ & 1.26 & 1.26 \\
\hline \multirow[t]{2}{*}{ GL (longitudinal) } & (pixels) & 50 & 80 \\
\hline & $(\mathrm{mm})$ & 2.1 & 3.4 \\
\hline \multirow[t]{2}{*}{ GL (transverse) } & (pixels) & 190 & 80 \\
\hline & $(\mathrm{mm})$ & 7.9 & 3.4 \\
\hline
\end{tabular}

The choice of the strain gage length (GL) depends on two constraints: firstly it has to be large enough to increase sensitivity for strain (Zone 2) and secondly small enough to represent accurately the strain gradients (Zone 1 ). In the Zone 2 we obtain a uniform strain field and the GL is chosen far from the sample boundaries. In addition, same gage lengths were selected axially and transversely in order to consider the same sensitivity in the two main directions $x$ and $y$. Applied to the image sequences, this method allows to calculate the evolution of the strain components and thus leads to the stress-strain curves.

In the Zone 1 and for a given state of stress, the correlation treatment gives access to the strain profile in the height of the central area investigated. Thus, if the axial GL is limited in order to describe the necking phenomenon, the transverse GL considers almost the entire width of the specimen to increase measurement sensitivity. Parameters used for the treatments are summarized in Table 2.

According to the GL definition, macro-homogeneous strain in Zone 2 or localized strains in Zone 1 are calculated as follows. The knowledge of cumulated displacements, until a given state of stress makes possible the evaluation of the strain tensor at the sample surface. The determination of the gradient tensor of transformation is based on local least-squares adjustment, using a linear fit of the displacement field around a considered point which is contained in the suitable Gage Length (Fig. 3(b)).

The two-dimensional displacement gradient tensor $\mathbf{F}$ is defined as

$$
\begin{aligned}
\boldsymbol{F} & =\mathbf{1}+\boldsymbol{G r a d} \vec{u}(\vec{x})=\left(\begin{array}{cc}
1+\frac{\partial u_{x}}{\partial x} & \frac{\partial u_{x}}{\partial y} \\
\frac{\partial u_{y}}{\partial x} & 1+\frac{\partial u_{y}}{\partial y}
\end{array}\right) \\
& =\left(\begin{array}{cc}
1+a_{x} & b_{x} \\
a_{y} & 1+b_{y}
\end{array}\right)
\end{aligned}
$$

where the boldface terms are related to tensors, $u_{x}$ and $u_{y}$ are the transverse and the longitudinal components of the cumulated displacement vector $\vec{u}=\left(u_{x}, u_{y}\right)$ which depends on the current position $\vec{x}=(x, y), a_{x}, b_{x}, a_{y}, b_{y}$ are constant parameters which define the linear approximation of the displacement field following:

$\left\{\begin{array}{l}u_{x}=a_{x} x+b_{x} y+c_{x} \\ u_{y}=a_{y} x+b_{y} y+c_{y}\end{array}\right.$

The calculation of the displacement gradient around the considered point allows the calculation of the Green-Lagrange strain tensor components as

$\boldsymbol{E}=\frac{1}{2}\left[\boldsymbol{F}^{T} \otimes \boldsymbol{F}-\mathbf{1}\right]$

where $\otimes$ is the tensor product, the superscript $T$ denotes the transpose operator and $\mathbf{1}$ is the identity tensor.
Correlation provides, all over the sample surface, the in-plane displacement and thus the Green-Lagrange strain tensor. From these Lagrangian strains, one can evaluate the logarithmic (or Hencky) strain tensor $\boldsymbol{E}_{L}$ as

$\boldsymbol{E}_{L}=\frac{1}{2} \ln \left(\boldsymbol{F}^{T} \otimes \boldsymbol{F}\right)=\frac{1}{2} \ln (2 \boldsymbol{E}+\mathbf{1})$

In the case of uniaxial tensile tests and for an elastic behaviour, extra diagonal terms of the logarithmic strain tensor are equal to zero. It yields to the in-plane logarithmic strain tensor components:

$$
\begin{aligned}
\boldsymbol{E}_{L} & =\left(\begin{array}{cc}
E_{L-x} & 0 \\
0 & E_{L-y}
\end{array}\right) \text { with }\left\{\begin{array}{l}
E_{L-x}=\frac{1}{2} \ln \left(2 E_{x x}+1\right) \\
E_{L-y}=\frac{1}{2} \ln \left(2 E_{y y}+1\right)
\end{array} \text { and } \boldsymbol{E}\right. \\
& =\left(\begin{array}{cc}
E_{x x} & E_{x y} \\
E_{x y} & E_{y y}
\end{array}\right)
\end{aligned}
$$

Logarithmic volumetric strain equals the sum of the logarithmic strains over the three axes. If we assume transverse isotropy for the material we can deduce that both transverse strains are equal. It yields to the expression of the logarithmic volumetric strain $E_{L-V \text { : }}$

$E_{L-V}=\ln \left(\frac{V}{V_{0}}\right)=2 E_{L-x}+E_{L-y}$

where $V$ is the current volume and $V_{0}$ is the initial volume.

From now on, the volumetric and longitudinal strains, respectively $E_{L-V}$ and $E_{L-y}$, refer to logarithmic strains which are additive.

Under very small strains the studied materials present an elastic behaviour and then the Green-Lagrange and the Hencky strain tensors are assumed to be equal. The analytical volumetric strain $E_{L-V-a n}$ can be defined as a function of both the measured Poisson's ratio $\nu$ and the longitudinal strain by the following expression:

$E_{L-V-a n}=(1-2 \nu) E_{L-y}$

2.2.2.4. Calibration. In this part, we want to assess the accuracy of the experimental displacements deduced from the DIC algorithm.

a . Evaluation of the out-of-plane displacement of the sample during a uniaxial tensile test

For the previous digital image correlation process we make the assumption of negligible out-of-plane displacements of the specimen

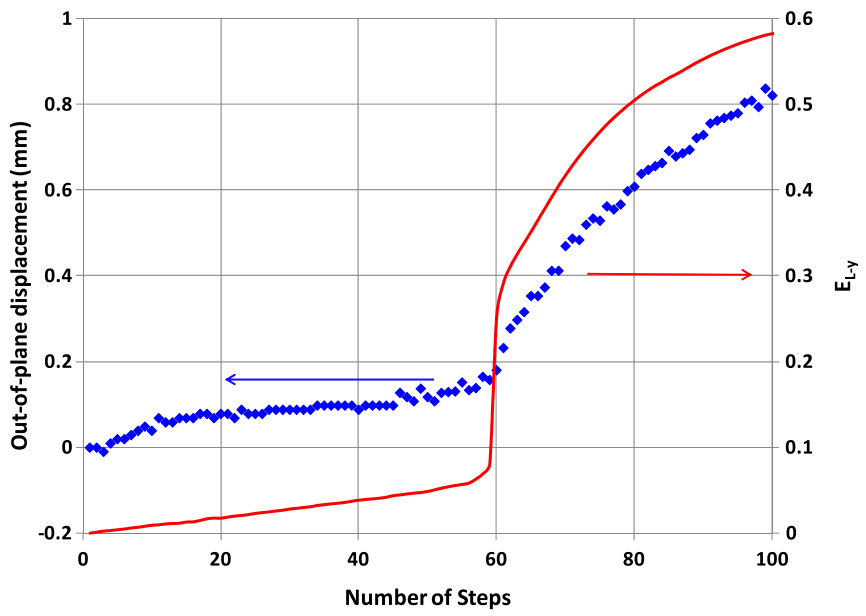

Fig. 4. Out-of-plane displacement and axial strain as a function of number of steps 
during the tensile test. However the specimen exhibits a Poisson's contraction associated with rigid body motion in the $z$ direction. These phenomena induce an error in the representation of the specimen in the coordinate system of the camera. This error has to be evaluated.

With an LVDT sensor located in contact with the specimen. In Fig. 4 we can follow the out-of-plane displacement vs. the number of steps on a tensile test conducted beyond the striction. The axial strain is obtained by the spatial correlation process presented in the previous paragraph. For this example, the maximal displacement never exceeds $1 \mathrm{~mm}$ at the centre of the specimen (zone 1) where necking is initiated. Two zones are clearly visible in the out-ofplane displacement curve: in the first one before the neck initiation, the out-of-plane displacement raises slowly as expected by the combination of a rigid body motion of the grips line and the elastic contraction (Poisson's effect). This stage is followed (from step 59), by an acceleration of the out-of-plane displacement rate as the neck occurred: this phenomenon induced by the plastic strain is explained by an accelerated rate of the axial strain accompanied by a sudden decrease of the cross section. By assuming a "tangent Poisson's ratio" of 0.5 (for an isochoric response associated to a plastic behaviour) and a locally measured axial strain $\left(E_{y y}\right)$ of 1.10 $\left(E_{L-y}=0.58\right)$, the out-of-plane displacement is estimated to be approximately $0.5 \mathrm{~mm}$ according to the thickness of the specimen $(e=4 \mathrm{~mm})$. This value does not seem too far from the measured value $(0.84 \mathrm{~mm})$, the difference is probably inherent to complementary more important rigid body movement of the grips in the $z$ direction. We can link analytically the error on the in-plane-strains $(\delta \epsilon)$ with the out-of-plane measured displacement $(\delta d)$.

$\delta \epsilon=G \frac{\delta d}{d}=\delta d \frac{l}{d^{2}}$

where $G$ is the optical magnification which is also the ratio between the distance lens/image $(l=55 \mathrm{~mm})$ and the distance lens/object $(d=680 \mathrm{~mm})$. On the basis of this function, the maximum of measured out-of-plane displacement leads to a relative error of less than $10^{-4}$ on the in-plane deformations. At the end of the elastic response (step 59), a relative error of $2.10^{-5}$ is to be compared to an axial strain $\left(E_{\mathrm{yy}}=810^{-2}\right)$. At the end of the test the relative error decreases since an error of $10.10^{-5}$ (with an out-of-plane displacement of $0.84 \mathrm{~mm}$ ) is obtained for an axial strain equal to $\left(E_{\mathrm{yy}}=11010^{-2}\right)$. So, in both cases the impact of this out-of-plane displacement on the strain value remains quasi-negligible.

\section{b . Calibration of the strain measurement}

Sources of error in the data obtained by the DIC procedures can be separated into noise and systematic components. Since these errors are not a function of the strain, a simple acquisition of one hundred images sequence was performed before starting the test [19]. The images were analysed with the same subset size (CS $=30$ pixels) than for DIC processing described in the Section 2.2.2.3. The error distribution of results obtained for the mesh correlation never exceeds 5/100 pixels and corresponds to a standard deviation (SD) of $10^{-2}$ pixel (as illustrated in Fig. 5 for $U_{x}$ displacement component). This random error is strongly a function of both the subset size and the quality of the image signature. Thus, a systematic error of $6 / 1000$ of pixel can be observed (given by the mean of the $U_{x}$ distribution in Fig. 5) and induces a slight shift off the $Y$-axis which has no effect on the evaluation of the strains.

The modeling of the cumulated error on the principal stretch ratios can be assessed by a normal law fitted according to the standard deviation obtained on displacements throughout two successive steps: firstly it consists in evaluating the cumulative error on the summed displacement components over a given

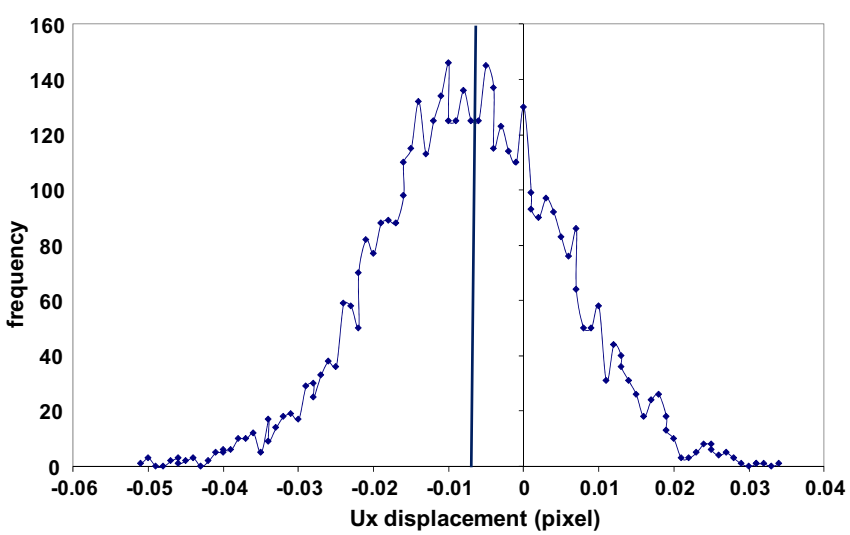

Fig. 5. Displacement uncertainty.

number of steps (20 for this work). This is achieved by generating a random sum of the standard deviation obtained on displacement in each point of a virtual mesh defined by the parameters of correlation shown in Table 2. Secondly, according to the post processing described in Section 2.2.2.3, the determination of the gradient tensor of transformation gives access to the cumulative error versus number of step. Considering both the resolution obtained previously on displacements ( $\mathrm{SD}=10^{-2}$ pixels) and the virtual mesh used in zones 1 and 2, the cumulative error does not exceed $10^{-4}$ and $10^{-6}$, respectively to the level of strain [13].

\section{Results and discussion}

\subsection{Macro-homogeneous study}

\subsubsection{Results}

Elastic properties, as both Young's modulus and Poisson's ratio, are obtained from stress-strain curves established in Zone 2 where the material response is macro-homogeneous. Stress comes from data acquired by the universal testing machine and the in-plane strains are obtained from DIC processing described in Table 2 . The experimental results displayed in Fig. 6 concern the mechanical behaviour of a PC specimen. Young's modulus (E) value is given by the slope, calculated between 0 and $1 \%$ strain of the curve giving axial stress vs. axial strain. Poisson's ratio $\nu$ equals the opposite of the ratio between the slopes of curves giving axial stress vs. respectively axial and transverse strains.

The Young's modulus values, for the different tested formulations, are shown in Table 3. The bracketed values beside modulus values describe the relative deviations with the PC's sample.

The incorporation of fillers, even at $1 \mathrm{wt} \%$, induces a rise of the Young's modulus. Fillers contribute to the reinforcement of the material. Regarding the valuesof Poisson's ratios, it have been observed no variability for all formulations compared to the PC's sample $(\nu=0.35)$.

\subsubsection{Micromechanical modeling}

Two continuum-based elastic micromechanical models are used to predict elastic properties of composites made of polycarbonate and silica or alumina nanoparticles for various interfaces. Both models are briefly described below.

It is assumed that the composite and each component follow an isotropic linear elastic constitutive law (Hooke's law) as follows:

$\sigma=C \epsilon$

where $\boldsymbol{\sigma}, \boldsymbol{C}$ and $\boldsymbol{\epsilon}$ are respectively the stress, the stiffness and the strain tensors. 


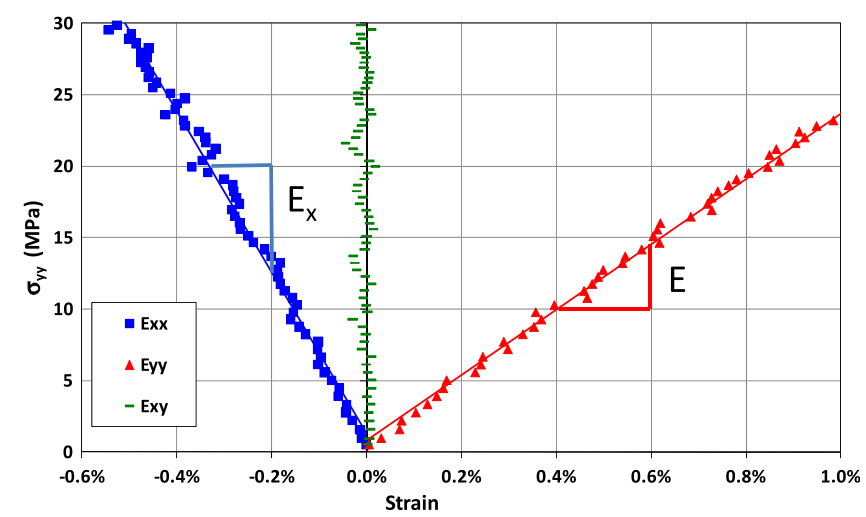

Fig. 6. Stress-strains curves obtained in the Zone 2 for tensile test performed on a PC specimen $\left(\nu=-\frac{E}{E_{X}}\right)$

The necessary data for the mechanical modeling are the following:

-PC: 2.4 GPa for the Young's modulus, 0.35 for the Poisson's ratio and $1200 \mathrm{~kg} \mathrm{~m}^{-3}$ for the density;

-Alumina Alu C: $390 \mathrm{GPa}$ for the Young's modulus, 0.25 for the Poisson's ratio and $3400 \mathrm{~kg} \mathrm{~m}^{-3}$ for the density;

- Silica A300: 73 GPa for the Young's modulus, 0.16 for the Poisson's ratio and $2200 \mathrm{~kg} \mathrm{~m}^{-3}$ for the density;

3.1.2.1. The Eshelby's model. The Eshelby's model has been already used to predict the elastic properties of two phase composites (matrix and particle) as a function of the particle volume fraction and geometry $[22,23]$. The particle is supposed to be ellipsoidal shaped. For this method, the overall stiffness tensor of the composite containing the isotropic components is:

$\boldsymbol{C}=\boldsymbol{C}_{m}+\boldsymbol{c}_{p}\left(\boldsymbol{C}_{p}-\boldsymbol{C}_{m}\right) \boldsymbol{T}_{p}$

where the subscripts $m$ or $p$ indicate respectively the matrix and the particle, $c$ is the volume fraction and $\boldsymbol{T}_{p}$ is the dilute strainconcentration tensor of the particles given by:

$\boldsymbol{T}_{p}=\left(\boldsymbol{I}+\boldsymbol{S}_{p}\left(\boldsymbol{C}_{m}\right)^{-1}\left(\boldsymbol{C}_{p}-\boldsymbol{C}_{m}\right)\right)^{-1}$

where $\boldsymbol{S}_{p}$ is the Eshelby's tensor (which depends on the Poisson's ratio of the matrix under the assumption of spherical particles, details can be found in [23]).

In this model, perfect bonding between particles and matrix and very low particle concentration are assumed and the composite contains only two phases. It is known that sizes of particle and interphase (which is a three dimensional region immediately surrounding the particle) influence the overall mechanical properties particularly in the case of nanocomposite materials [24,25].

3.1.2.2. The dilute coated-inclusion model. Some authors [26-28] built up models to predict elastic properties of composites with particles which exhibit an interphase of the same spherical symmetry as the particle. Interphase is assumed to have a constant thickness. For this model, called "dilute coated-inclusion model", the stiffness tensor is:

$\boldsymbol{C}=\boldsymbol{C}_{m}+\boldsymbol{c}_{p}\left(\boldsymbol{C}_{p}-\boldsymbol{C}_{m}\right) \boldsymbol{T}_{p}+\boldsymbol{c}_{i}\left(\boldsymbol{C}_{i}-\boldsymbol{C}_{m}\right) \boldsymbol{T}_{i}$,

where the subscript $\mathrm{i}$ indicates the interphase and $\boldsymbol{T}_{p}, \boldsymbol{T}_{i}$ are the dilute strain-concentration tensors (respectively of the particle and the interphase). These tensors depend on the Poisson's ratio of the matrix, the radius of the spherical particles and the interface thickness (details of calculation can be found in [27]).

3.1.2.3. Comparison between model and experiments. A comparison between the Eshelby's model, the dilute coated-inclusion model and experimental results is shown in Table 3 by considering variations of the interphase properties. Firstly, a parametric study was carried out as a function of the interphase elastic modulus (and thickness), varying such value between that of the matrix and that of the particles. We opted for an interphase with material properties of the particle (alumina and silica). This choice was arbitrary. It certainly induces an overestimation of the homogenized Young's modulus. Indeed, we do not intend to achieve the determination of both the thickness and the stiffness of the interphase. We intend, preferably, to highlight the existence of the interphase as well as a methodology of characterization when microstructural data are available (for instance the thickness).

The dilute coated model applied with an interphase's thickness of $67 \%$ (respectively $41 \%$ ) of the inclusion's radius in the case of alumina (respectively silica) conducts to the best fit with experimental results. The mean diameter of the particles is respectively $7 \mathrm{~nm}$ for silica (A300) and $13 \mathrm{~nm}$ for alumina (AluC). The lack of available data on the interfacial zone leads, in a first approximation, to consider a homogenous interphase. Nevertheless, some authors consider nanoparticles surrounded by interfaces with graded properties [29].

As shown in Table 3 classical 2-phases models (as Eshelby's model) under-estimate the Young's modulus of the composites. So models commonly used for the description of composites with micro-sized inclusions are not suitable for nanocomposites. As a matter of fact, the extensive interaction area between particles and matrix is widely under-estimated. It is now commonly accepted that the polymer close to the particle may have a different mechanical behaviour than the polymer far from the particle. So 3phases models, as shown in Table 3, are more able to predict accurate elastic properties by an adequate choice of interphase thickness and mechanical properties. Our analysis could be improved by a better characterization of the interphase (principally geometry and properties).

\subsection{Local study of the necking phenomenon}

Strain profiles along the specimen height and through the necking area (Zone 1 ) have to be determined for different times (corresponding to different states of stress) (Fig. 7(a)). Each curve of the Fig. 7(a) is represented by a mark (open losange) on the Load/ time curve in Fig. 7(b). Parameters used for the DIC processing are

Table 3

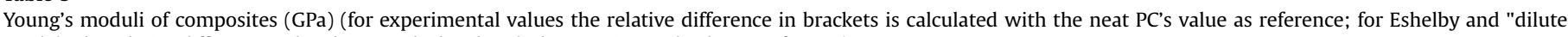
model" the relative difference in brackets is calculated with the experimental value as reference).

\begin{tabular}{|c|c|c|c|c|c|c|}
\hline & PC/Alumina $1 \mathrm{wt} \%$ & PC/Alumina 3 wt\% & PC/Silica 1 wt\% & PC/Silica 3 wt $\%$ & PC & Standard deviation \\
\hline Experimental & $2.4(+4.3 \%)$ & $2.6(+13.0 \%)$ & $2.4(+4.2 \%)$ & $2.6(+13.3 \%)$ & 2.3 & \pm 0.05 \\
\hline Eshelby & $2.3(-4.2 \%)$ & $2.4(-7.7 \%)$ & $2.4(0 \%)$ & $2.4(-7.7 \%)$ & & \\
\hline Dilute model & $2.4(0 \%)$ & $2.6(0 \%)$ & $2.4(0 \%)$ & $2.6(0 \%)$ & & \\
\hline
\end{tabular}



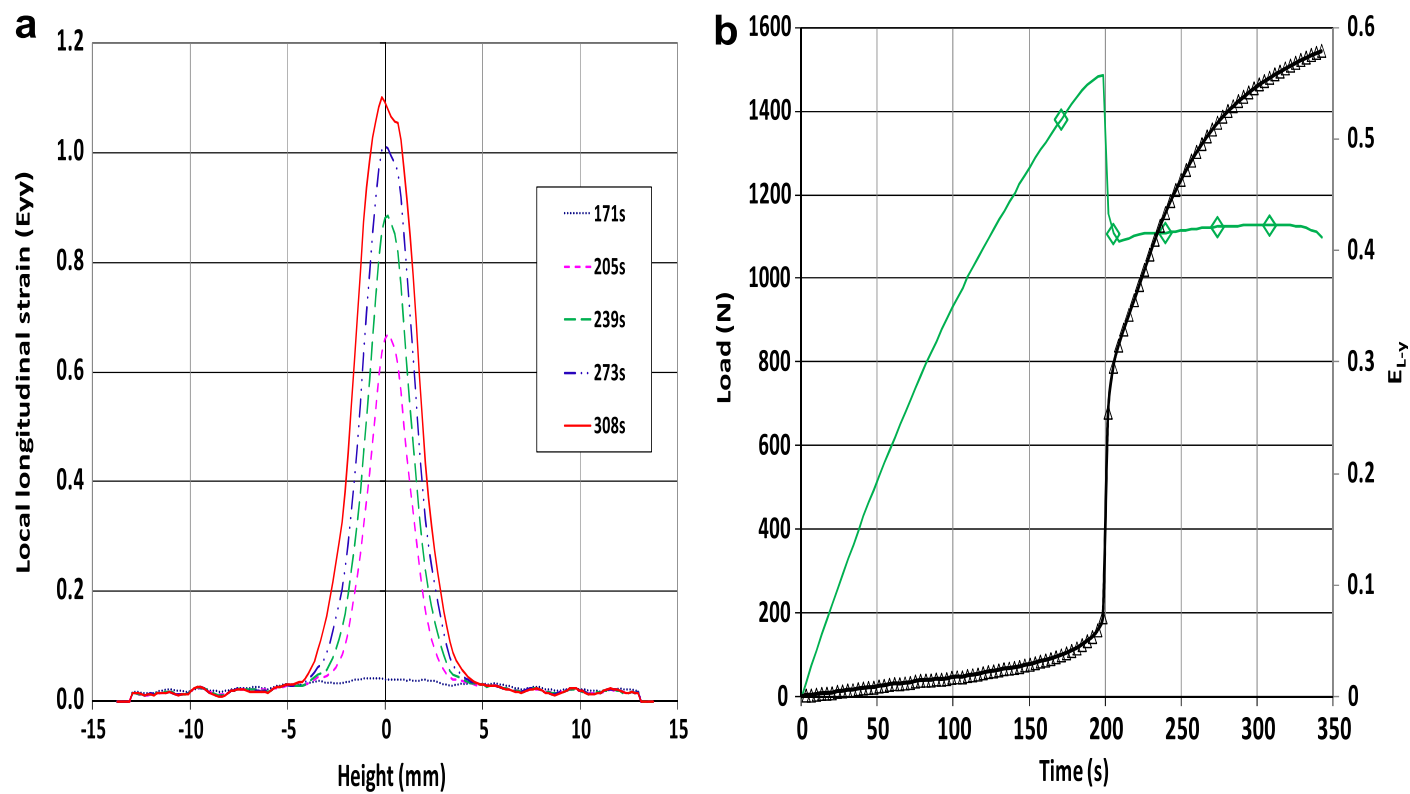

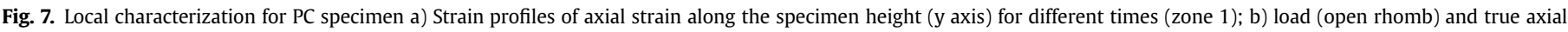
strain (open triangle) as a function of time.

summarized in Table 2. The experimental results displayed here concern the mechanical behaviour of a PC specimen during a standardized uniaxial tensile test (according to ISO 527). It is worth noticing that profiles obtained after the yield stress seem to be centred at the location of the reduced section (zone 1). If observed at a more local scale, a slight shift of the profile is inherent to the resolution of the mesh $(0.21 \mathrm{~mm}$ between two consecutive points). Moreover we can notice the significant increase of longitudinal strain in the necked zone with respect to points outside. These first results illustrate then an interesting feature of the digital extensometry, that is the local strain measurement: the absence of predefined markers drawn on the sample ensures the exact location of the necking point and allows to monitor its strain evolution. Moreover, this approach allows following the extension of the necking area and providing an interesting analysis of its shape described below.

If the shape of these profiles will be discussed later regarding increasing of the area of damage, we may, at first, extract the evolution of the nominal stress as a function of longitudinal logarithmic strain for the point which presents the maximum strain (Fig. 8). Effectively, as mentioned by other authors, Polycarbonate

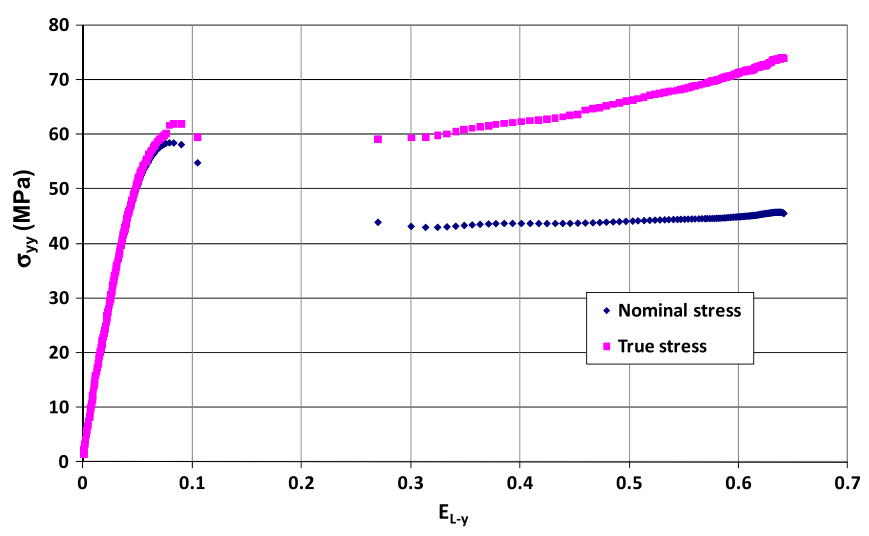

Fig. 8. Axial stress vs. axial strain in the necking area for neat PC. presents a ductile behaviour characterized by flow localization [19]. On these curves, the evolution of the nominal stress is characterized by a lack of points just beyond the yield point. This phenomenon, which is simultaneous with the neck formation, corresponds to a sudden acceleration of the axial strain in the necking zone. As an illustration of this phenomenon, the load and the local strain are plotted vs time in Fig. 7(b): The local strain increases monotonically with the load and when necking occurs, a drastic drop of the load is observed simultaneously with the rise of the local strain. Before the yield point, the material is supposed to present a homogeneous elastic behaviour.

The definition of the nominal stress neglects the cross-sectional area reduction occurring during the necking. Considering this variation, the true axial stress $\sigma_{t}$ has also been evaluated. It can be calculated from the nominal stress $\sigma_{N}$ as:

$\sigma_{t}=\frac{F}{S_{\text {true }}}=\sigma_{N} \exp \left(-2 E_{L-x}\right)$

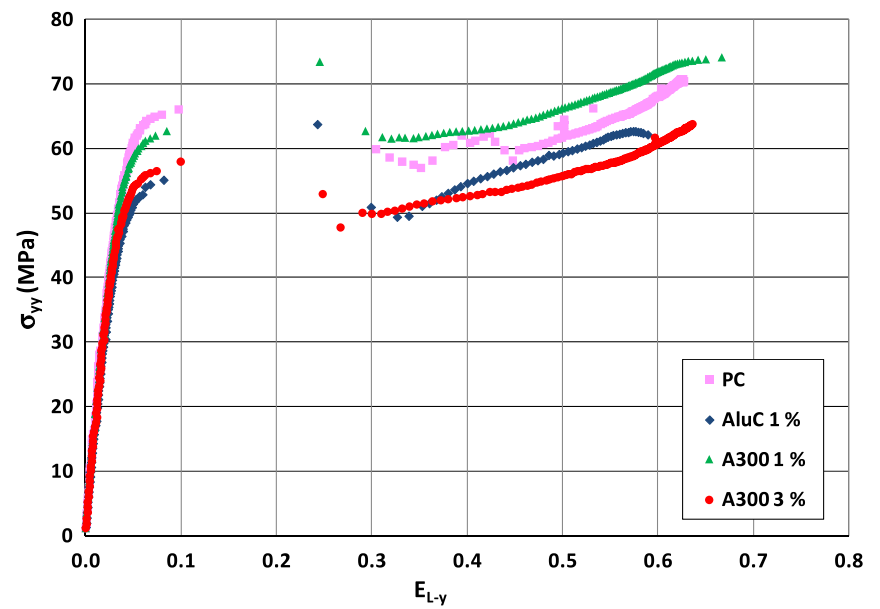

Fig. 9. Macroscopic true stress as function of strain for PC with silica or alumina fillers at different levels of incorporation (static tensile tests). 

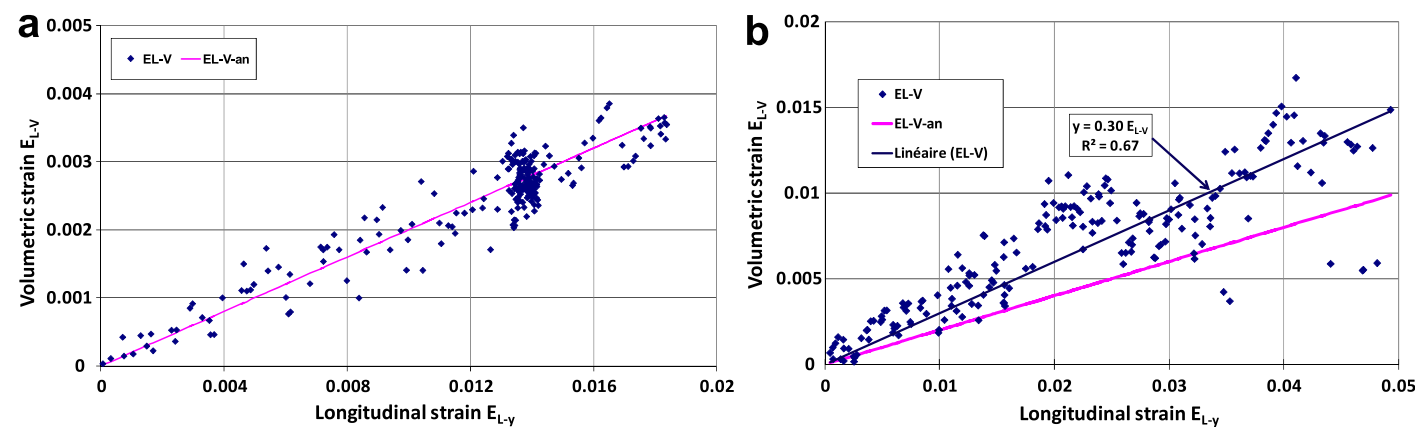

Fig. 10. Volumetric strain vs. axial strain (a) zone 2, (b) zone 1 for a tensile test performed on a Alu C 1 wt \% specimen.

where $S_{\text {true }}$ is the current cross-sectional area of the specimen. For this calculation, it was assumed that the specimen deforms isotropically. It can be seen that the large yield trough displayed on the $\sigma_{N}$ vs. $E_{L-x}$ curve nearly disappears when the data are converted to $\sigma_{t}$ vs. $E_{L-x}$ form. As the neck propagates, the nominal stress remains constant whereas the true stress increases continuously to fairly high values at failure, which indicates that the material exhibits a strain-hardening phenomenon. This strain-hardening is very well known in glassy polymers (PS, PMMA ...) [30] and supposed to be due to polymer chains alignment under tension.

The addition of fillers gives the typical true stress-strain curves shown in Fig. 9. As fracture was observed just after the yield stress for the formulation containing $3 \mathrm{wt} \%$ alumina, this formulation was not included in the local study of necking. For others formulations, the stress reached before necking decreases significantly with incorporation of fillers. It should be noted that the decrease in yield stress is more significant for formulation with alumina fillers compared to others formulations. In the same way, it seems that $1 \mathrm{wt} \%$ silica leads to an increase of the true stress after necking. These results are indicative of disruption of the polymer molecular structure and will be discussed below.

\subsection{Evolution of the volumetric strain at local scale}

\subsubsection{Volumetric strain in the elastic domain}

Given that all the tested samples show the same general volumetric strain evolution, only one representative curve will be presented. The aim of this section is to focus on the evolution of the volumetric strain in the elastic domain that is to say at low strain, before yield point. As previously mentioned, the volumetric strain has been calculated both in Zone 2 and in Zone 1 where the necking is initiated and the strain is maximal.

Fig. 10 presents the evolution of the measured and analytical volumetric strain as a function of the longitudinal strain in the zones 1 and 2 at low strain and for the same tensile test performed on a PC specimen.

In Zone 2, the volumetric strain measured by photomechanics corresponds to the analytical elastic response. This means that the elastic behaviour, assuming hypothesis of transverse isotropy, allows describing the volumetric strain undergone by the material in the Zone 2.

The values of the volumetric strain at the central point (Fig. 10(b)) are also in good agreement with the analytical volumetric strain. The difference between the analytical and experimental volumetric strains in the central zone could be attributed to two phenomena: firstly the error on the strain measurement inherent to the calculation mode (Table 2), which is, in this case, associated to a small GL. The baseline accuracy of the local strain measurement is thus about $\pm 3300 \mu \epsilon$ which has to be compared to the range of deviation of $\pm 260 \mu \epsilon$ obtained on the macro- homogeneous strain in Zone 2 (Fig. 10(a)). Secondly, the range of local strain plotted on the Fig. 10(b), between 0 and 0.05 , reaches values for which the behaviour is no longer purely elastic. Despite a lack of resolution, the parameters set used for the correlation processing in Zone 1 allow describing locally the strain state.

\subsubsection{Volumetric strain at the central point before and after the yield point}

Fig. 11 illustrates the evolution of the volumetric strain during the tensile test for each formulation.

After yield point (at 0.05 strain level), a significant volumetric contraction is observed. This phenomenon was already observed by different authors [19,20,31], and several explanations are given:

- Elastic recovery as the stress level drops during the strain softening stage of deformation (after yield point) [19]

- Macromolecular orientation due to the onset of plastic deformation (could be confirmed by X-ray diffraction), this phenomenon occurring essentially into glassy polymers (such as PC) $[20,31]$

It can be noticed that this phenomenon concerns only a short stage of the deformation ranging between 0.05 and 0.3 .

The propagation of the necking is characterized by an increase in the volumetric strain slope. This phenomenon could reveal the damage of the material. After this stage, curves of volumetric strain vs. axial or transversal strains reach a maximum before to saturate. This corresponds to the propagation of the necking out of the observation area. This phenomenon already observed, is explained by the stabilization of the strains within the neck area whereas the

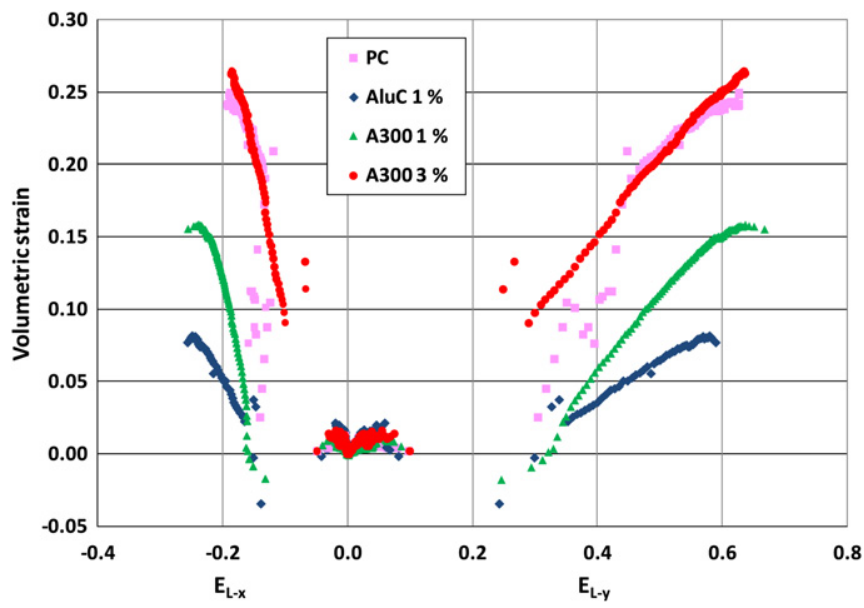

Fig. 11. Volumetric strain vs. axial strain $\mathrm{E}_{\mathrm{L}-\mathrm{y}}$ and transverse strain $\mathrm{E}_{\mathrm{L}-\mathrm{x}}$. 


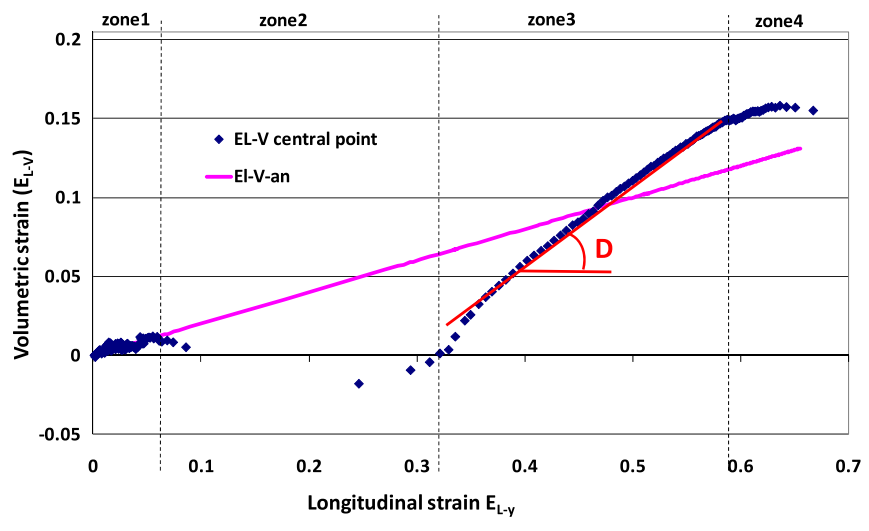

Fig. 12. Volumetric strain vs. axial strain during a tensile test performed on A300 1\% specimen and definition of damage parameter.

neck fronts stretch up to the extremities of the specimen [32]. Thus, the observation of these curves allows identifying four stages during the tensile test: the first one is an elastic domain before yield point and where the strain is homogeneous within the observed area; in stage 2, a localisation phenomenon occurs at yield point corresponding to the plastic instability and the initiation of the necking. The decrease in volume could be due to elastic recovery as the load level drops (Fig. 8(b)) [19]. This stage is followed by the propagation of damage in the material during necking propagation (stage 3). Finally, a pseudo-elastic behaviour of the material is observed in stage 4 after the propagation of the necking out of the observation area. As an illustration of these stages, the volumetric strain is plotted in Fig. 12 as a function of time in the case of a tensile test performed on a A300 1\% specimen. It is worth noting that the low resolution on strain components in Zone 1 (associated to the small GL used) leads to a sufficient relative resolution as the volumetric strain increases.

An estimation of the damage during the necking propagation (stage 3 ) undergone by each material is evaluated by calculating the slope of the curve giving volumetric strain vs. axial strain according to the following expression (Fig. 12):

$D=\left.\frac{\partial E_{L-V}}{\partial E_{L-y}}\right|_{E L-y}$

To consider the plastic theory and overcome the strain-rate sensitivity of the damage, an estimation of the strain rate after contraction phase (during the necking propagation) for three formulations validates that strain-rate remains in the same order

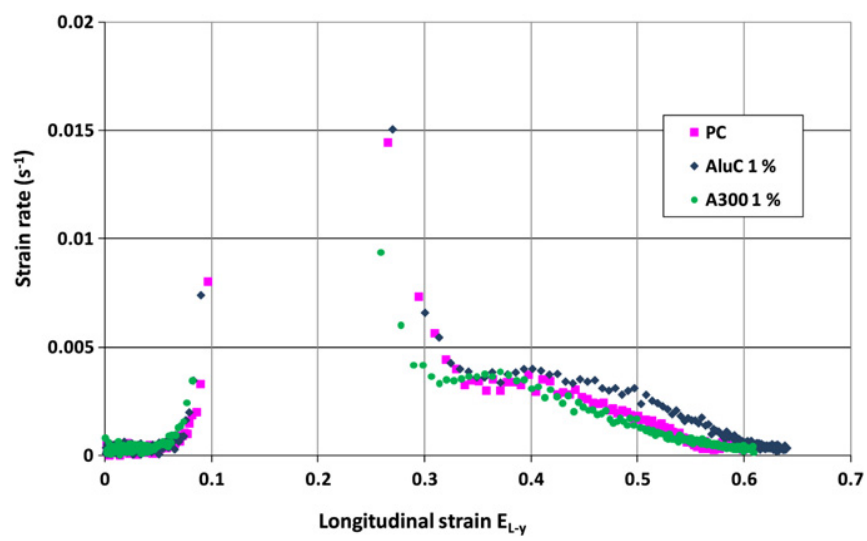

Fig. 13. Axial strain-rate vs. axial strain at the central point during the tensile test for each formulation.

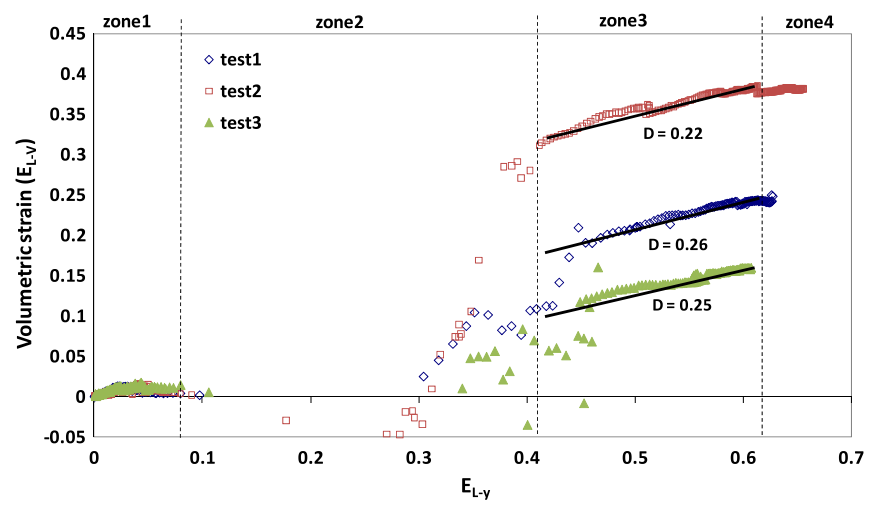

Fig. 14. Volumetric strain vs. axial strain during three tensile test performed on PC specimen and estimation of the damage parameter.

for the axial deformation ranging between 0.3 and 0.5 corresponding to the stage 3 (Fig. 13). This point is important to assess quantitatively a description of flow behaviour and to calculate the associated coefficients [33]. It may be noted also that, the strainrate is quite the same for all samples in the whole range of strain: this is important because it allows comparison with damages assessed for each material. As described before, the relatively low frequency of the image recordings $(0.5 \mathrm{~Hz})$ associated with the sudden acceleration of the elongations at the necking, conducts to the lack of points at the strain ranges from $E_{L-y}=0.1$ to $E_{L-y}=0.25$.

Three tensile tests are performed with neat PC and the volumetric-strain curves vs. true axial strain are drawn on Fig. 14. It is worth noting that, the increase of the volumetric strain in stage 3 leads to similar damage values for all three tests. Also, it should be noted the lack of reproducibility of the neck initiation at the second stage: this observation seems important because it determines the choice of the behavior modeling when the material response becomes non-homogeneous. After the first stage, where the specimen deforms homogeneously, the strain rises quickly when once necking begins and the local response of the material leads to major discrepancies in the evolution of the volumetric strain. It is interesting to observe that, whatever the level of volumetric strain reached at the end of the second stage, the propagation of necking leads to the same evolution of volumetric strain.

Table 4 presents values of the damage parameter $\mathrm{D}$ for the different tested materials. As observed Fig. 11, the incorporation of alumina fillers induces a significant decrease in the level of the volumetric strain on one hand, and a slower rate of change on the other. The calculation of the volumetric neglects shear strains and assumes an isotropic strain in the transverse cross-section. Despite these assumptions, the damage parameter appears to be similar for both samples with silica fillers associated with a much higher level of volumetric strain than others nano-charged formulations. This phenomenon is probably related to a higher dissipative potential of strain (plasticity, damage) in the formulation with silica fillers. The observation of the necking zone by SEM demonstrates the influence of the fillers on the morphology of the necking and on the damaging mechanisms (Fig. 15). This figure shows the distribution of the silica and alumina nanoparticles in the PC matrix (Fig. 15(b)). Both types of nanoparticles appear more or less aggregated. For A300 silica the dispersion is quite homogeneous and aggregates

Table 4

damage parameters vs formulation.

\begin{tabular}{lllll}
\hline & PC & PC/alumina 1 wt\% & PC/silica 1 wt\% & PC/silica 3 wt\% \\
\hline D & $0.24 \pm 0.02$ & 0.26 & 0.42 & 0.50 \\
\hline
\end{tabular}


sizes are less than $80 \mathrm{~nm}$. Concerning Alu C alumina, the dispersion is more heterogeneous: presence of small aggregates about $100 \mathrm{~nm}$ size and few bigger aggregates (about $1 \mu \mathrm{m}$ size). Moreover, the numerous huge crazes observed on the neat PC sample (Fig. 15(a)) shows that crazing is the main damaging mechanism for PC. It is generally observed that addition of fillers reduces the damage. The number of crazes is far less important for the $1 \mathrm{wt} \%$ alumina filled specimen. In this specimen, decohesion at the matrix-particle's interface has been observed (Fig. 15(c)) and seems to be the main damaging mechanism. The incorporation of silica leads to a more homogeneous damage of the material which presents small and numerous crazes all over the surface of the necking zone. Decohesion phenomena have also been observed at interfaces between particles and matrix. The more homogeneous damage observed for silica fillers can be correlated to the idea of a larger dissipative potential of strain explained above. For silica fillers, damage (cavitation) is shown to depend on the relative percentage of this component. A similar result was obtained by Yang et al. [34] for $\mathrm{HDPE} / \mathrm{CaCO}_{3}$ blends.

\subsection{Statistical study of the necking}

As mentioned previously, image correlation was applied to Zone 2 and to Zone 1, where the necking is initiated. The central point corresponds to the point where the strain is the highest. Then a strain profile could be established along the tensile bar as illustrated in Fig. 16(a). We aim to characterize the degree of flatness of each distribution; As Kurtosis coefficient conducts to differentiate

\begin{tabular}{lccc}
\hline a Surface & b $\begin{array}{c}\text { Fillers } \\
\text { dispersion }\end{array}$ & C & Fracture \\
$\underline{200 \mu \mathrm{m}}$ & $\underline{10 \mu \mathrm{m}}$
\end{tabular}
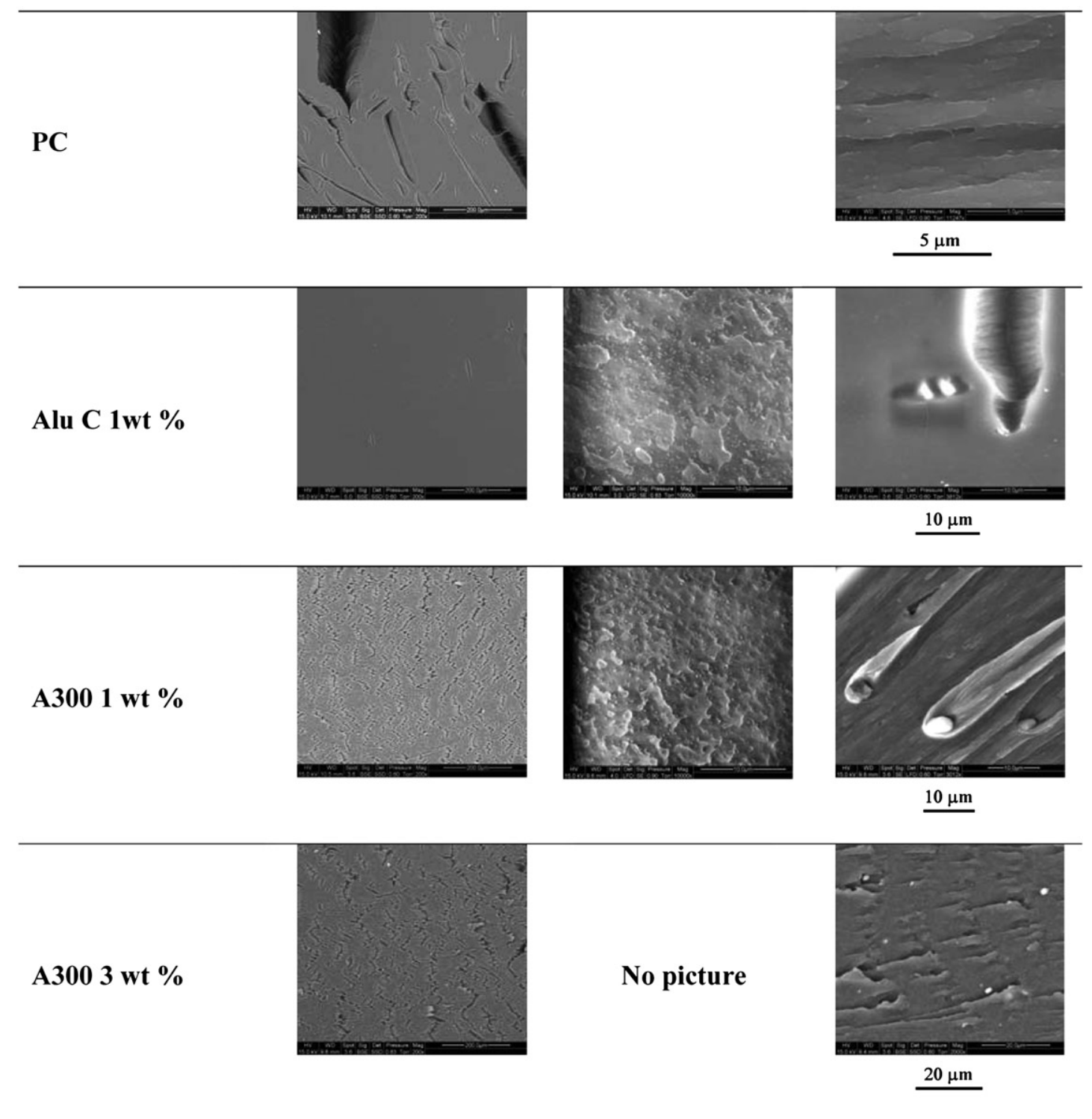

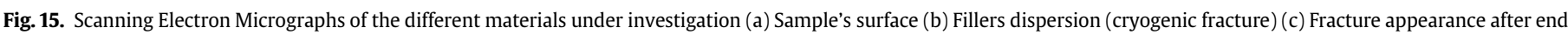
of tensile test. 
a
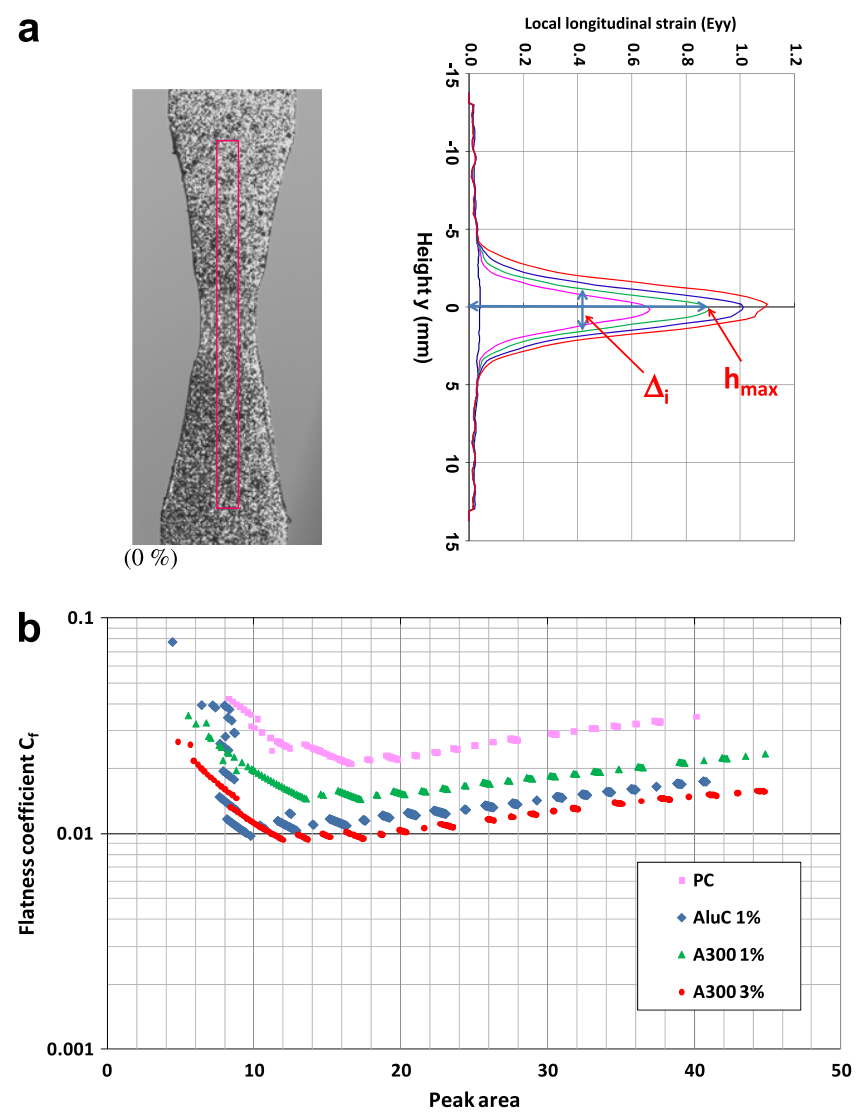

Fig. 16. Strain profile distribution (a) Flatness coefficient as a function of peak area (b).

a sharp peak from a flat-topped in a non gaussian curve [33], we opt for a normalized coefficient which is the ratio between $\Delta i$ the peak width at half-height and $h_{\max }$ the total height (see Fig. 16(a)). We introduce a statistical parameter, called flatness coefficient $\left(C_{f}\right)$, defined as follows:

$$
C_{f}=\frac{\left(\frac{\Delta i}{h_{\max }}\right)}{\left.\left(\frac{\Delta i}{h_{\max }}\right)\right|_{\sigma_{y}}}
$$

where $\sigma_{y}$ refers to the stress at yield point, that is to say the stress corresponding to the beginning of the necking. This coefficient traduces the ability of the necking zone to spread over the tensile bar or to be localized in the central zone. It can be related to the shape of the strain profile. In fact, the higher the flattening coefficient, the wider the strain peak and the more uniform the necking zone. On the contrary, the lower the flattening coefficient, the narrower the strain peak and the more localized the necking zone.

The evolution of the flattening coefficient as a function of the strain peak area is presented on the Fig. 16(b) for the different tested materials.

The general evolution is the same for all the tested materials. The $C_{f}$ shows a drop that corresponds to the beginning of the necking and the apparition of the strain peak; then, the $C_{f}$ stabilizes during the neck propagation. Although the neck was initiated at the minimal cross-section in the centre part of the sample, the crosssection area decreases as the necking fronts stretch of along the longitudinal axis. This phenomenon promotes the rupture by thinning of the cross-section in the central part. Despite of this specificity related to the geometry of the sample, which complicates the necking analysis, notches were similarly machined in the centre part of all samples, and comparisons are allowed between the different formulations. The influence of the formulation on the necking morphology is obvious. $C_{f}$ decreases significantly with the incorporation of fillers. The strain peak of these materials is narrower than in the case of the unfilled PC. Necking seems to be more localized for alumina formulations compared to silica formulations. The lack of dissipative phenomenon could explained the early fracture before the yield point for the AluC 3\%. It seems that the presence of fillers in the PC matrix prevents the necking from propagation. This result is in total agreement with the observation of the necking by SEM presented in the previous section.

\section{Conclusion}

A methodology was established for assessing the phenomenological behaviour of polycarbonate nanocomposites reinforced by alumina or silica nanoparticles at low levels of incorporation. The experimental technique is based on a non-contact and nonintrusive digital extensometry, already widely used, the Digital Image Correlation (DIC). The correlation parameters have been optimized and calibrated to establish the mechanical response of the material in two areas of the tensile bar, in the central zone where strain localization phenomena takes place, and in the nonmachined zone. The elastic properties were particularly calculated in the non-machined zone far away from local instabilities. Beside this macro-homogeneous approach, the local strain measurements provide an interesting tool to follow the extension of the necking area.

Concerning the elastic behaviour, Young's modulus evalutation based on the macro-homogeneous approach has been compared with two continuum-based elastic micromechanical models, the Eshelby's model and the dilute coated-inclusion model: the incorporation of fillers, even at $1 \mathrm{wt} \%$, induces a slight rise of the Young's modulus. Otherwise, it has been observed no variability of the Poisson's ratios compared to the neat PC's sample.

At a local scale, the propagation of the necking is characterized by a sudden increase of the volumetric strain. Also, it has been observed a stochastic response in terms of volumetric strain just after the neck initiation: this observation reveals the choice of the modeling when the material response becomes non-homogeneous. Experimental and modeling works are produced in CMGD/EMA laboratory to take account of this specific aspect. The local approach gave also a comparative study between the different formulations and highlighted the influence of the incorporation of fillers on the material behaviour. In particular the flatness coefficient provides a good parameter to describe the evolution of axial profile strains through the neck: a relatively low flatness coefficient qualifies a rupture by thinning of cross-section at the initiating area of the neck, while a high coefficient indicates the stretching of necking fronts along the longitudinal axis. If the first case is generally observed for all formulations with additional fillers compared to neat $\mathrm{PC}$, thinning phenomenon seems to be observed more significantly for alumina formulations compared to silica formulations. This point should probably explained also why fracture appeared early and without plasticity for the formulation with the higher rate of alumina fillers.

These results indicate that extent of the plastic damage is controlled by the shape of the necking whose growth contributes to the dissipative potential of the material before fracture.

\section{References}

[1] Rong MZ, Zhang MQ, Zheng YX, Zeng HM, Walter R, Friedrich K. Polymer 2001;42(1):167-83.

[2] Zhao Y, Schiraldi DA. Polymer 2005;46(25):11640-7. 
[3] Carrión FJ, Sanes J, Bermúdez MD. Wear 2007;262(11-12):1504-10.

4] Jia X, Ling X. Wear 2005;258(9):1342-7.

[5] Yoon PJ, Hunter DL, Paul DR. Polymer 2003;44(18):5341-54.

[6] Wang Z, Xie G, Wang X, Li G, Zhang Z. Materials Letters 2006;60(8):1035-8.

[7] Pötschke P, Fornes TD, Paul DR. Polymer 2002;43(11):3247-55.

[8] Pötschke P, Abdel-Goad M, Alig I, Dudkin S, Lellinger D. Polymer 2004;45(26): $8863-70$.

[9] Abdel-Goad M, Potschke P. Journal of Non-Newtonian Fluid Mechanics 2005; 128(1):2-6.

[10] Yoon PJ, Hunter DL, Paul DR. Polymer 2003;44(18):5323-39.

[11] Hasegawa N, Okamoto H, Kato M, Usuki A, Sato N. Polymer 2003;44(10): 2933-7.

[12] Lazzeri A, Zebarjad SM, Pracella M, Cavalier K, Rosa R. Polymer 2005;46(3): 827-44.

[13] Laraba-Abbes F, Ienny P, Piques R. Polymer 2003;44(3):807-20.

[14] Foulc MP, Bergeret A, Ferry L, Ienny P, Crespy A. Polymer Degradation and Stability 2005;89(3):461-70.

[15] Satapathy BK, Weidisch R, Pötschke P, Janke A. Composites Science and Technology 2007;67(5):867-79.

[16] Kramer EJ, Berger LL. Craze growth and fracture. In: Kaush HH, editor Advances in Polymer Science, Crazing in polymers. Berlin-Heidelberg, Germany: Springer Verlag; 1990. p. 1-68.

[17] Ienny P, Caro-Bretelle AS, Pagnacco E. European Journal of Computational Mechanics 2009;18(3-4):353-76.

[18] Avril S, Bonnet M, Bretelle AS, Grédiac M, Hild F, Ienny P, et al. Experimental Mechanics 2008;48(4):381-402.

[19] Parsons E, Boyce MC, Parks DM. Polymer 2004;45(8):2665-84.
[20] G'Sell C, Hiver JM, Dahoun A. International Journal of Solids and Structures 2002;39(13-14):3857-72.

[21] G’Sell C, Dahoun A, Hiver JM, Addiego F. Proceedings "Matériaux 2002". Dijon France; 2002.

[22] Eshelby JD. The continuum theory of lattice defects. In: Seitz F, Turnbull D, editors. Solid State Physics, vol. 3. New York: Academic Press Inc.; 1956. p. 79-156.

[23] Eshelby JD. Proceedings of the Royal Society 1957;241(1226):376-96.

[24] Petrovic ZS, Cho YJ, Javni I, Magonov S, Yerina N, Schaefer DW, et al. Polyme 2004;45(12):4285-95

[25] Berriot J, Lequeux F, Monnerie L, Montes H, Long D, Sotta P. Journal of NonCrystalline Solids 2002;307-310:719-24.

[26] Hervé E, Zaoui A. European Journal of Mechanics - A/Solids 1990;9(6): 505-15.

[27] Hervé E, Zaoui A. International Journal of Engineering Science 1993;31(1):1-10.

[28] Marcadon V, Hervé E, Zaoui A. International Journal of Solids and Structures 2007;44(25-26):8213-28.

[29] Sevostianov I, Kachanov M. International Journal of Solids and Structures 2007;44(3-4):1304-15.

[30] Li HX, Buckley CP. International Journal of Solids and Structures 2009;46: 1607-23.

[31] Addiego F, Dahoun A, G'Sell C, Hiver JM. In: Oil \& Gas Science and Technology, 61(6). Revue de l'Institut français du pétrole; 2006. p. 715-24.

[32] Rinaldi R, Gaertner R, Brunet M, Chazeau L, Vidal-Sallé E, Gauthier C. International Journal of Non-Linear Mechanics 2010;46(3):507-18.

[33] G'Sell C, Jonas JJ. Journal of Materials Science 1979;14:583-91.

34] Yang YL, Bai SL, G'Sell C, Hiver JM. Polymer Engineering and Science 2006 46(11):1512-22. 